\title{
On Continuous Selection Sets of Non-Lipschitzian Quantum Stochastic Evolution Inclusions
}

\author{
Sheila Bishop \\ Department of Mathematics, Covenant University, KM 10 Idiroko Road, PMB 1023, Ota, Ogun State, Nigeria \\ Correspondence should be addressed to Sheila Bishop; sheila.bishop@covenantuniversity.edu.ng
}

Received 25 March 2015; Accepted 7 July 2015

Academic Editor: Alexander M. Krasnosel'skii

Copyright (C) 2015 Sheila Bishop. This is an open access article distributed under the Creative Commons Attribution License, which permits unrestricted use, distribution, and reproduction in any medium, provided the original work is properly cited.

We establish existence of a continuous selection of multifunctions associated with quantum stochastic evolution inclusions under a general Lipschitz condition. The coefficients here are multifunctions but not necessarily Lipschitz.

\section{Introduction}

We consider the following Cauchy problem defined in [1]:

$$
\begin{array}{r}
d X(t) \in\left(E_{2}(t, X(t)) d \wedge_{\pi}(t)+F_{2}(t, X(t)) d A_{g}(t)\right. \\
\left.+G_{2}(t, X(t)) d A_{f^{+}}(t)+H_{2}(t, X(t)) d t\right) \\
-\left(E_{1}(t, X(t)) d \wedge_{\pi}(t)+F_{1}(t, X(t)) d A_{g}(t)\right. \\
\left.+G_{1}(t, X(t)) d A_{f^{+}}(t)+H_{1}(t, X(t)) d t\right) \\
X(0)=a, t \in[0, T],
\end{array}
$$

where $E_{1}, F_{1}, G_{1}$, and $H_{1}$ are hypermaximal monotone multivalued maps and $E_{2}, F_{2}, G_{2}$, and $H_{2}$ are multifunctions but not necessarily Lipschitz.

As observed by [2], problems of continuous selection, features of reachable sets, and the solution sets of classical differential inclusions have attracted considerable attention [3-6]. The existence and nonuniqueness of solutions of such inclusions have been investigated to a large extent. See [7] and the references therein.

Existence of continuous selections of multifunctions associated with the sets of solutions of Lipschitzian and non-Lipschitzian quantum stochastic differential inclusions (QSDIs) has been considered in $[2,8]$, while the existence of solution of quantum stochastic evolution arising from hypermaximal monotone coefficients was established in [9].
Also in $[10,11]$ several results have been established concerning some properties of the solution sets of QSDIs. Results concerning the topological properties of solution sets of Lipschitzian QSDI were also considered in [12]. In [1], results on continuous selections of solution sets of quantum stochastic evolution inclusions (QSEIs) were established under the Lipschitz condition defined in $[2,13]$.

In order to generalize the results in the literature concerning QSDI, in [8] existence of continuous selections of solutions sets of non-Lipschitzian quantum stochastic differential inclusions was considered. It was proved that certain inclusion problems do not necessarily satisfy the Lipschitz condition defined in $[2,13]$. In [8], the map $x \rightarrow P(t, x)(\eta, \xi)$ satisfied a general Lipschitz condition with values that are closed but not necessarily convex or bounded subsets of the field of complex numbers. This work is concerned with similar results established in [8] where the coefficients are not necessarily Lipschitz. The results here generalize existing results in the literature [1] concerning quantum stochastic evolution inclusions (QSEIs).

The rest of this paper is organized as follows: in Section 2, we present the foundations for establishing the major results. In Section 3, we will establish the major results. Our method will be a blend of the methods applied in $[1,8]$.

\section{Preliminaries}

All through this work, as in $[2,8]$, we adopt the definitions and notations of the following spaces, subspaces, and sets: 
$\mathbb{R} \otimes \Gamma\left(L_{\gamma}^{2} \mathbb{R}_{+}\right), \operatorname{Ad}(\widetilde{\mathscr{A}}), \operatorname{Ad}(\widetilde{\mathscr{A}})_{\mathrm{wac}}, L_{\mathrm{loc}}^{p}(\widetilde{\mathscr{A}}),\left(\operatorname{Ad}(\widetilde{\mathscr{A}})_{\mathrm{wac}}, \tau_{w}\right)$, $L^{1}(I, \mathbb{D} \otimes \mathbb{E}), C(I, \mathbb{D} \otimes \mathbb{E})$, and $L_{r, \mathrm{Loc}}^{\infty}\left(\mathbb{R}_{+}\right)$for a fixed Hilbert space $r . \tau_{1}, \tau_{c}, \widetilde{\mathscr{A}}, \operatorname{clos}(S),($ resp., $\operatorname{Comp}(S)), \operatorname{clos}(\widetilde{\mathscr{A}}), \rho(A, B)$, $\operatorname{clos}(\mathbb{C}), a_{k}, a_{\eta \xi, k}, \Phi_{\eta \xi, k}(\cdot)$, and so forth. For the completion of the space $\left(\operatorname{Ad}(\widetilde{\mathscr{A}})_{\mathrm{wac}}, \tau^{\mathrm{wac}}\right)$ and the generated topology and many more we refer the reader to $[2,8]$.

For the definitions of lower semicontinuous multivalued map (l.s.c.) $\Phi: S \rightarrow 2^{\bar{A}}$, measurability and measurability of a multifunction $(t, x) \rightarrow \Phi(t, x)(\eta, \xi)$, decomposable subset of a space, and locally absolutely $P$-integrable and adapted multivalued stochastic process $\Phi$, we refer the reader to $[2,8]$.

We consider the following quantum stochastic differential inclusion (QSDI) defined in [2]:

$$
\begin{aligned}
& d X(t) \in\left(E(t, X(t)) d \wedge_{\pi}(t)+F(t, X(t)) d A_{g}(t)\right. \\
& \left.+G(t, X(t)) d A_{f^{+}}(t)+H(t, X(t)) d t\right), \\
& X(0)=a, \text { almost all } t \in I=[0, T],
\end{aligned}
$$

where the multivalued stochastic processes $E, F, G, H \in$ $L_{\mathrm{loc}}^{2}(I \times \widetilde{\mathscr{A}})_{\mathrm{mvs}}$ and $(a, 0) \in I \times \widetilde{\mathscr{A}}$ is fixed. The equivalent form of inclusion (1.2) established in [13] is given by

$$
\begin{aligned}
& \frac{d}{d t}\langle\eta, X(t) \xi\rangle \in P(t, X)(\eta, \xi) \\
& X\left(t_{0}\right)=X_{0}, t \in\left[t_{0}, T\right] .
\end{aligned}
$$

Inclusion (2) is understood in the sense of Hudson and Parthasarathy [14] while inclusion (3) is a first order nonclassical ordinary differential inclusion with a sesquilinear form valued map $P$ as the right-hand side. For existence of solution of inclusion (3) and the explicit form of the map $(t, X) \rightarrow P(t, X)(\eta, \xi)$ appearing in inclusion (3) see [13] and also see [7] for nonuniqueness of solution of (3). We employ the locally convex topological $(\widetilde{\mathscr{A}})$ space of noncommutative stochastic processes defined in [13].

In this work, we consider the following evolution problem given by

$$
\begin{array}{r}
\frac{d}{d t}\langle\eta, X(t) \xi\rangle \in P_{2}(t, X)(\eta, \xi)-P_{1}(t, X)(\eta, \xi) \\
X(0)=a, t \in[0, T],
\end{array}
$$

where the sesquilinear form valued map $P_{1}: I \times \widetilde{\mathscr{A}} \rightarrow$ $2^{\operatorname{sesq}(\mathbb{D} \otimes \mathbb{E})^{2}}$ is hypermaximal monotone and the sesquilinear form valued map $P_{2}: I \times \widetilde{\mathscr{A}} \rightarrow 2^{\operatorname{sesq}(\mathbb{D} \otimes \mathbb{E})^{2}}$ satisfies a general Lipschitz condition defined in [8]. The point $a$ ranges in a subset $A$ of $\widetilde{\mathscr{A}}$ such that the set $A(\eta, \xi)=\langle\eta, a \xi\rangle: a \in A$ is compact in $\mathbb{C}$.

Motivated by the result in [8], we extend the results in [1], to a class of evolution inclusion that depends on a more general Lipschitz condition $W(t) \neq t$. Hence the results here are weaker than the results in [1].

Let $P_{1}$ be hypermaximal monotone, and the map $(t, x) \rightarrow$ $P_{2}(t, x)(\eta, \xi)$ appearing in (4) is assumed to satisfy the following conditions with $W(t) \neq t$ :
(1) The map $(t, x) \in \rightarrow P_{2}(t, x)(\eta, \xi)$ is measurable.

(2) There exists a map $K_{\eta \xi}^{p}:[0, T] \rightarrow \mathbb{R}_{+}$lying in $L_{\text {loc }}^{I}([0$, $T])$ and the function $W:[0, \infty) \rightarrow \mathbb{R}$ increasing and continuous with $W(0)=0$ such that

$$
\begin{gathered}
\rho\left(P_{2}(t, x)(\eta, \xi)-P_{2}(t, y)(\eta, \xi)\right) \\
\leq K_{\eta \xi}^{p}(t) W\left(\|x-y\|_{\eta \xi}\right),
\end{gathered}
$$

a.e., $t \in[0, T]$, and for each pair $x, y \in \widetilde{\mathscr{A}}$.

(3) There exists a $\beta$ which lies in $L_{\text {loc }}^{1}([0, T])$ such that

$$
d\left(0, P_{2}(t, 0)(\eta, \xi)\right) \leq \beta_{\eta \xi}, \quad \text { a.e., } t \in I .
$$

For the map $P: I \times \widetilde{\mathscr{A}} \rightarrow 2^{\operatorname{sesq}(\mathbb{D} \otimes \mathbb{E})^{2}}$, define the map $\langle\eta, a \xi\rangle \rightarrow \phi_{P}\left(a_{\eta \xi}\right)$ by

$$
\begin{gathered}
\phi_{P}\left(a_{\eta \xi}\right)=\left\{v_{\eta \xi} \in L^{1}(I, \mathbb{D} \otimes \mathbb{E}): v_{\eta \xi}\right. \\
\in P(t, a)(\eta, \xi), \text { a.e., } t \in I\} .
\end{gathered}
$$

Remark 1. Conditions (1) and (3) are similar to conditions $S_{(i)}$ and $S_{(i i i)}$ in [1], while condition $S_{(i i)}$ has been modified to accommodate the general Lipschitz condition defined above. If we take $W(t)=t$, then condition (2) reduces to condition $S_{(i i)}$ in [1].

We adopt the proof of the following results established in [1] since the proof of these results is independent of the Lipschitz function.

Lemma 2. Consider the multivalued stochastic process $P: I \times$ $\widetilde{\mathscr{A}} \rightarrow 2^{\text {sesq }(\mathbb{D} \otimes \mathbb{E})^{2}}$, and assume that

(i) the map $(t, x) \rightarrow P(t, x)(\eta, \xi)$ is measurable,

(ii) the $\operatorname{map}(t, \cdot) \rightarrow P(t, \cdot)(\eta, \xi)$ is l.s.c.

Then the map $\phi_{P}$ given by (7) is lower semicontinuous (l.s.c) from $A(\eta, \xi)$ into $\mathscr{D}$ if and only if there exists a continuous map $\beta: A(\eta, \xi) \rightarrow L^{1}(I, \mathbb{R})$ such that, for every $a \in A, a_{\eta \xi} \equiv$ $\langle\eta, a \xi\rangle \in A(\eta, \xi)$,

$$
d(0, P(t, a)(\eta, \xi)) \leq \beta\left(a_{\eta \xi}\right)(t), \quad \text { a.e., } t \in I .
$$

Lemma 3. Let the multivalued stochastic process $\phi$ : $A(\eta, \xi) \rightarrow \mathscr{D}$ be l.s.c. Assume that

(i) $\varphi: A(\eta, \xi) \rightarrow L^{1}(I, \mathbb{D} \otimes \mathbb{E})$ and $\phi: A(\eta, \xi) \rightarrow L^{1}(I$, $\mathbb{R})$ are continuous,

(ii) for every $a_{\eta \xi} \in A(\eta, \xi)$ the set $\Phi\left(a_{\eta \xi}\right)$ defined by (10) in [1] is nonempty.

Then the multivalued stochastic process $\phi: A(\eta, \xi) \rightarrow \mathscr{D}$ is l.s.c. and therefore admits a continuous selection. 
For $a \in \overline{D\left(P_{1}(t, \cdot)(\eta, \xi)\right)}$ and $p \in L^{1}(I, \mathbb{D} \underline{\mathbb{E}})$, we consider the Cauchy problem

$$
\begin{aligned}
&\left(H_{p}\right) \frac{d}{d t}\langle\eta, X(t) \xi\rangle \in\langle\eta, p(t) \xi\rangle \\
&-P_{1}(t, X(t))(\eta, \xi), \\
& X(0)=a .
\end{aligned}
$$

For the existence of a unique weak solution of the Cauchy problem $\left(H_{p}\right)$ see [15]. We adopt definition 2.1 concerning the solution of $\left(H_{p}\right)$ and remark 2.1 all in [1]. Hence condition (11) in [1] follows.

Let $P_{2}: I \times \widetilde{\mathscr{A}} \rightarrow 2^{\text {sesq(}(\mathbb{D} \otimes \mathbb{E})^{2}}$ satisfy conditions (1)-(3). Consider the Cauchy problem

$$
\begin{aligned}
&\left(H_{a}\right) \frac{d}{d t}\langle\eta, X(t) \xi\rangle \in P_{2}(t, X(t))(\eta, \xi) \\
&-P_{1}(t, X(t))(\eta, \xi), \\
& X(0)=a,
\end{aligned}
$$

where $a \in A_{0}$.

Definition 4. A function $x(\cdot, a): I \rightarrow \widetilde{A}$ is called a solution of $\left(H_{a}\right)$ if there exists $\langle\eta, p(t) \xi\rangle \in L^{1}(I$, sesq $(\mathbb{D} \otimes \mathbb{E}))$, a selection of $P_{2}(\cdot, x(\cdot, a))(\eta, \xi)$ such that $x(\cdot, a)$ is a weak solution of the Cauchy problem $H_{p(\cdot a)}$.

We denote by $S_{T}(a)$ the set of all solutions of $\left(H_{a}\right)$ and prove a continuous selection theorem from the map $S_{T}(a)(\eta, \xi)$, where

$$
S_{T}(a)(\eta, \xi)=\langle\eta, \Phi \xi\rangle \backslash \Phi \in S_{T}(a) .
$$

Just as in [8], an important consequence of our main result is that the set map $\langle\eta, a \xi\rangle \rightarrow S_{T}(a)(\eta, \xi)$ can be continuously represented in the form

$$
g(\langle\eta, a \xi\rangle, u)=S_{T}(a)(\eta, \xi)
$$

with the Lipschitz condition $W(t) \neq t$. See Corollaries 3.2 and 3.3 in [2]. This generalizes all results in the literature established under the Lipschitz condition $W(t)=t$.

\section{Major Results}

In this section, we present our major results under the general Lipschitz condition defined above. We will establish the result by employing similar argument employed in the proof of Theorems 3.1 in [1] and 3.1 in [8] by highlighting the major changes due to condition (iii).

Theorem 5. Assume that the maps $P_{1}, P_{2}: I \times \widetilde{\mathscr{A}} \rightarrow$ $2^{\text {sesq }(\mathbb{D} \otimes \mathbb{E})^{2}}$ satisfy the following conditions:

(i) $P_{1}$ is hypermaximal monotone.

(ii) $(t, x) \rightarrow P_{2}(t, x)(\eta, \xi)$ is measurable. (iii) There exists a map $K_{\eta \xi}:[0, T] \rightarrow \mathbb{R}_{+}$lying in $L_{l o c}^{1}([0$, $T])$, such that

$$
\begin{aligned}
& \rho\left(P_{2}(t, x)(\eta, \xi), P_{2}(t, y)(\eta, \xi)\right) \\
& \leq K_{\eta \xi}(t) W\|x-y\|_{\eta \xi}
\end{aligned}
$$

a.e. in $I$, where $W(t) \neq t$.

(iv) There exists $\beta \in L_{l o c}^{1}(I)$ such that

$$
d\left(0, P_{2}(t, 0)(\eta, \xi)\right) \leq \beta_{\eta \xi}, \quad \text { a.e., } t \in I .
$$

If $A_{0} \subset \widetilde{\mathscr{A}}$, then there exists an adapted stochastic process $x$ : $I \times A_{0} \rightarrow \operatorname{sesq}(\mathbb{D} \otimes \mathbb{E})$ such that

(i) $\langle\eta, x(\cdot, a) \xi\rangle \in S_{T}(a)(\eta, \xi)$ for every $a \in A_{0}$;

(ii) $\langle\eta, a \xi\rangle \rightarrow\langle\eta, x(\cdot, a) \xi\rangle$ is continuous from $A_{0}(\eta, \xi)$ to $C(I, \mathbb{D} \otimes \mathbb{E})$.

Proof. Let $a \in A_{0}$ and $x_{0}(\cdot, a): I \rightarrow \widetilde{A}$ be the unique weak solution of the Cauchy problem

$$
\frac{d}{d t}\langle\eta, X(t) \xi\rangle \in-P_{1}(t, X(t))(\eta, \xi), \quad X(0)=a .
$$

For $K_{\eta \xi}$ and $\beta$ defined by (iii) and (iv), we define $b: A_{0}(\eta$, $\xi) \rightarrow L_{\text {loc }}^{1}(I)$ by

$$
b\left(a_{\eta \xi}\right)(t)=\beta_{\eta \xi}(t)+K_{\eta \xi}(t) W\left(\left\|x_{0}(t, a)\right\|_{\eta \xi}\right) .
$$

By remark 2.1 in [1], the map $\langle\eta, a \xi\rangle \rightarrow\left\langle\eta, x_{0}(\cdot, a) \xi\right\rangle$ is weakly continuous from $A_{0}(\eta, \xi)$ to $C(I, \mathbb{D} \otimes \mathbb{E})$. Hence from (16), it follows that $b(\cdot)$ is continuous from $\overline{A_{0}}(\eta, \xi)$ to $L_{\text {loc }}^{1}(I)$. And we have

$$
d\left(0, P_{2}\left(t, x_{0}(t, a)\right)(\eta, \xi)\right) \leq b\left(a_{\eta \xi}\right)(t), \quad \text { a.e. in } I
$$

for each $a_{\eta \xi} \in A_{0}(\eta, \xi)$.

As in [8] we fix $\epsilon>0$ and set $\epsilon=\epsilon / 2^{n+1}, n \in \mathbb{N}$. Define $\Phi_{0}: A_{0}(\eta, \xi) \rightarrow 2^{L(I, \mathbb{Q} \otimes \mathbb{E})}$ and $\phi_{0}: A_{0}(\eta, \xi) \rightarrow 2^{L(I, \mathbb{D} \otimes \mathbb{E})}$ by (14) and (15) in [1]. Using (16) and Lemma $2, \phi_{0}(\cdot)$ is lower semicontinuous (l.s.c.) and for each $a_{\eta, \xi} \in A_{0}(\eta, \xi)$, $\phi_{0}\left(a_{\eta \xi}\right) \neq 0$, and $W(t) \neq t$. Again by Lemma 3, there exists $\varphi_{0}(\eta, \xi) \rightarrow L(I, \mathbb{D} \otimes \mathbb{E})$, a continuous selection of $\phi_{0}(\cdot)$. Set $p_{0}(t, a)(\eta, \xi)=\varphi_{0}\left(a_{\eta \xi}\right)(t)$ as in $[1]$; then $p_{0}(\cdot, a)(\eta, \xi)$ is continuous, $p_{0}(t, a)(\eta, \xi) \in P_{2}(t, a)(\eta, \xi)$, and

$$
\left|p_{0}(t, a)(\eta, \xi)\right| \leq b\left(a_{\eta \xi}\right)(t)+\epsilon_{0}, \quad \text { a.e. } t \in I .
$$

If we set $M_{\eta \xi}=\int_{0}^{t} K_{\eta \xi}(s) d s$, where $a_{\eta \xi}$ is as defined, then, for each $a \in A_{0}$, we can define $\beta_{n}\left(a_{\eta \xi}\right)(t), n \geq 1$ as follows:

$$
\begin{aligned}
\beta_{n}\left(a_{\eta \xi}\right)(t)= & \int_{0}^{t}\left(a_{\eta \xi}\right)(s) \frac{\left[M_{\eta \xi}(t)-M_{\eta \xi}(s)\right]^{n-1} d s}{(n-1) !} \\
& +W T\left(\sum_{i=0}^{n} \epsilon_{i}\right) \frac{\left[M_{\eta \xi}(t)\right]^{n-1}}{(n-1) !}, \quad t \in I .
\end{aligned}
$$


Thus by (19) $\beta_{n}(\cdot)$ is continuous from $A_{0}(\eta, \xi)$ to $L_{\text {loc }}^{1}(I, \mathbb{R})$ since $b(\cdot)$ is continuous. Now if $x_{1}(\cdot, a): I \rightarrow \widetilde{\mathscr{A}}$ is the unique solution of the Cauchy problem

$$
\begin{array}{r}
\frac{d}{d t}\langle\eta, X(t) \xi\rangle \in\left\langle\eta, p_{0}(t) \xi\right\rangle-P_{1}(t, X(t))(\eta, \xi), \\
X(0)=a
\end{array}
$$

then, by (11) in [1], we have

$$
\begin{gathered}
\left|\left\langle\eta, x_{1}(t) \xi\right\rangle-\left\langle\eta, x_{0}(t) \xi\right\rangle\right| \leq \int_{0}^{t}\left|p_{0}(s, a)(\eta, \xi)\right| d s \\
\leq\left(\int_{0}^{t} b\left(a_{\eta \xi}\right)(s) d s+\epsilon_{0} T\right)<\beta_{1}\left(a_{\eta \xi}\right)(t),
\end{gathered}
$$

for each $a_{\eta \xi} \in A_{0}(\eta, \xi)$ and $t \in I \backslash\{0\}$. Now set $\left\langle\eta, p_{n}(s, a) \xi\right\rangle \equiv$ $p_{n}(s, a)(\eta, \xi)$ and assume that there exist sequences $\left\{p_{n}(\cdot\right.$, a) $\}_{n \in \mathbb{N}}$ and $\left\{x_{n}(\cdot, a)\right\}_{n \in \mathbb{N}}$ such that, for each $n \geq 1$, (a), (b), and (d) in [1] hold in this case while (c) becomes

$$
\begin{array}{r}
\left|\left\langle\eta, p_{1}(t, a) \xi\right\rangle-\left\langle\eta, p_{n-1}(t, a) \xi\right\rangle\right| \leq W K_{\eta \xi}(t) \beta_{n}(t), \\
\text { a.e. } t \in I,
\end{array}
$$

where $W$ is due to the Lipschitz function $K$. We now obtain the following by (22) and (11) in [1], $t \in I \backslash\{0\}$

$$
\begin{aligned}
& \left|\left\langle\eta, x_{n+1}(t, a) \xi\right\rangle-\left\langle\eta, x_{n}(t, a) \xi\right\rangle\right| \\
& \leq \int_{0}^{t}\left|\left\langle p_{n}(s, a)(\eta, \xi)\right\rangle-\left\langle p_{n-1}(s, a)(\eta, \xi)\right\rangle\right| d s \\
& \leq W \int_{0}^{t} K_{\eta \xi}(s) \beta_{n}\left(a_{\eta \xi}\right)(s) d s \\
& =W\left[\int_{0}^{t} b\left(a_{\eta \xi}\right)(s) \frac{\left[M_{\eta \xi}(t)-M_{\eta \xi}(s)\right]^{n}}{n !} d s\right] \\
& \quad+W T\left(\sum_{i=0}^{n} \epsilon_{i}\right) \frac{\left[M_{\eta \xi}(t)\right]^{n}}{n !}<W\left(\beta_{1}\left(a_{\eta \xi}\right)(t)\right) .
\end{aligned}
$$

Since $P$ is maximal monotone and hence hypermaximal monotone, we get

$$
\begin{aligned}
d & \left(\langle\eta, p(t, a) \xi\rangle, P_{2}\left(t, x_{n+1}(t, a)\right)(\eta, \xi)\right) \\
& \leq K_{\eta \xi}(t) W\left(\left\|x_{n+1}(t, a)-x_{n}(t, a)\right\|_{\eta \xi}\right) \\
& <K_{\eta \xi}(t) W\left(\beta_{n+1}\left(a_{\eta \xi}\right)(t)\right) .
\end{aligned}
$$

By (24) and Lemma 2, the multivalued map $\phi_{n+1}$ : $A_{0}(\eta, \xi) \rightarrow 2^{L^{1}(I, \mathbb{D} \otimes \mathbb{E})}$ defined by (19) in [1] is l.s.c. with decomposable closed nonempty values. Then by Lemma 3 , the sesquilinear form valued map $\varphi_{n+1}\left(a_{\eta \xi}\right)(t)$ still admits a continuous selection of $\Phi_{n+1}(\cdot)$.
If we set $\left\langle\eta, p_{n+1}(t, a) \xi\right\rangle=\varphi_{n+1}\left(a_{\eta \xi}\right)(t)$ for $a_{\eta \xi} \in A_{0}(\eta, \xi)$, $t \in I$, we have that $p_{n+1}$ satisfies the properties (a), (b) in [1] and (22); hence by (24), we obtain

$$
\begin{aligned}
& \Phi_{n+1}\left(a_{\eta \xi}\right)=\operatorname{cl}\left\{v_{\eta \xi}\right. \\
& \quad \in \phi_{n+1}\left(a_{\eta \xi}\right):\left|v_{\eta \xi}-\left\langle\eta, p_{n k}(t, a) \xi\right\rangle\right| \\
& \left.\quad<K_{\eta \xi}(t) W\left(\beta_{n+1}\left(a_{\eta \xi}\right)(t)\right)\right\}, \quad t \in I .
\end{aligned}
$$

Again by (22) and (23), we have

$$
\begin{aligned}
\left|\left\langle\eta, p_{n}(\cdot, a) \xi\right\rangle-\left\langle\eta, p_{n-1}(\cdot, a) \xi\right\rangle\right| \\
=\int_{0}^{T}\left|\left\langle p_{n}(s, a)(\eta, \xi)\right\rangle-\left\langle p_{n-1}(s, a)(\eta, \xi)\right\rangle\right| d s \\
\leq W\left[\int_{0}^{T} b\left(a_{\eta \xi}\right)(s) \frac{\left[M_{\eta \xi}(T)-M_{\eta \xi}(s)\right]^{n}}{n !} d s\right] \\
\quad+W T\left(\sum_{i=0}^{n} \epsilon_{i}\right) \frac{\left[M_{\eta \xi}(T)\right]^{n}}{n !} \\
\leq W\left(\frac{\left[\left\|K_{\eta \xi}(t)\right\|_{1}\right]^{n}}{n !}\left(\left\|b\left(a_{\eta \xi}\right)\right\|+T \epsilon\right)\right) .
\end{aligned}
$$

Since $a_{\eta \xi} \rightarrow\left\|b\left(a_{\eta \xi}\right)(t)\right\|$ is continuous, then it is locally bounded. It follows by (26) that the sequence $\left\{p_{n}(\cdot, a)\right\}_{n \in \mathbb{N}}$ satisfies the Cauchy condition uniformly. If $p(\cdot, a)$ is the limit of the given sequence, then $a_{\eta \xi} \rightarrow\langle\eta, p(\cdot, a) \xi\rangle$ is also weakly continuous from $A_{0}(\eta, \xi)$ into $L^{1}(I, \mathbb{D} \otimes \mathbb{E})$.

Now if we use (23) and (26), we get

$$
\begin{aligned}
& \left|\left\langle\eta, x_{n+1}(\cdot, a) \xi\right\rangle-\left\langle\eta, x_{n}(\cdot, a) \xi\right\rangle\right| \\
& \quad \leq\left|\left\langle\eta, p_{n}(\cdot, a) \xi\right\rangle-\left\langle\eta, p_{n-1}(\cdot, a) \xi\right\rangle\right| \\
& \quad \leq \frac{W\left[\left\|K_{\eta \xi}(t)\right\|_{1}\right]^{n}}{n !}\left(\left\|b\left(a_{\eta \xi}\right)\right\|_{1}+T \epsilon\right) .
\end{aligned}
$$

Hence $\left\{\left\langle\eta, x_{n}(\cdot, a) \xi\right\rangle\right\}$ is Cauchy in $C(I, \mathbb{D} \otimes \mathbb{E})$ with respect to $a$. Then the map $a_{\eta \xi} \rightarrow\left\langle\eta, x_{n}(\cdot, a) \xi\right\rangle$ is weakly continuous from $A_{0}(\eta$,$) to C(I, \mathbb{D} \otimes \mathbb{E})$ and so also the map $\left\langle\eta, x_{n}(\cdot, a) \xi\right\rangle \rightarrow\langle\eta, x(\cdot, a) \xi\rangle$ uniformly and

$$
\begin{aligned}
& d\left(\left\langle\eta, p_{n}(t, a) \xi\right\rangle, P(t, x(t, a))(\eta, \xi)\right) \\
& \quad \leq K_{\eta \xi}(t) W\left(\left\|x_{n}(\cdot, a)-x(\cdot, a)\right\|_{\eta \xi}\right) .
\end{aligned}
$$

Therefore, the result (22) in [1] holds here. If we let $p_{0}=p$ and $x_{1}(\cdot, a)$ be the unique weak solution of the Cauchy problem (20), we obtain by (11) in [1]

$$
\begin{aligned}
& \left|\left\langle\eta, x_{n}(t, a) \xi\right\rangle-\left\langle\eta, x_{1}(t, a) \xi\right\rangle\right| \\
& \quad \leq \int_{0}^{t}\left|\left\langle\eta, p_{n}(s, a) \xi\right\rangle-\langle\eta, p(s, a) \xi\rangle\right| d s .
\end{aligned}
$$


If $n \rightarrow \infty$, then $\left\langle\eta, x_{1}(\cdot, a) \xi\right\rangle \equiv\langle\eta, x(\cdot, a) \xi\rangle$. Therefore, $x(\cdot, a)$ is the weak solution of (20), and the result

$$
\langle\eta, x(\cdot, a) \xi\rangle \in S_{T}(a)(\eta, \xi)
$$

holds here under the general Lipschitz condition.

\section{Conflict of Interests}

The author declares that there is no conflict of interests.

\section{References}

[1] M. O. Ogundiran, "Continuous selections of solution sets of quantum stochastic evolution inclusion," Journal of Nonlinear Functional Analysis, vol. 2014, article 4, 2014.

[2] E. O. Ayoola, "Continuous selections of solution sets of lipschitzian quantum stochastic differential inclusions," International Journal of Theoretical Physics, vol. 43, no. 10, pp. 20412059, 2004.

[3] A. Cellina, "On the set of solutions to Lipschitzian differential inclusions," Differential and Integral Equations, vol. 1, no. 4, pp. 495-500, 1988.

[4] J.-P. Aubin and A. Cellina, Differential Inclusions, Springer, Berlin, Germany, 1984.

[5] A. F. Filippov, "Classical solutions of differential equations with multi-valued right-hand side," SIAM Journal on Control and Optimization, vol. 5, pp. 609-621, 1967.

[6] M. Kisielewicz, Differential Inclusions and Optimal Control, vol. 44 of Mathematics and Its Applications (East European Series), Kluwer Academic Publishers, Dordrecht, The Netherlands, 1991.

[7] E. O. Ayoola, "Further results on the existence of continuous selections of solution sets of quantum stochastic differential inclusions," Dynamic Systems and Applications, vol. 17, no. 3-4, pp. 609-624, 2008.

[8] S. A. Bishop and T. A. Anake, "Extension of continuous selection sets to non-lipschitzian quantum stochastic differential inclusion," Stochastic Analysis and Applications, vol. 31, no. 6, pp. 1114-1124, 2013.

[9] G. O. Ekhaguere, "Quantum stochastic differential inclusions of hypermaximal monotone type," International Journal of Theoretical Physics, vol. 34, no. 3, pp. 323-353, 1995.

[10] E. O. Ayoola, "Construction of approximate attainability sets for Lipschitzian quantum stochastic differential inclusions," Stochastic Analysis and Applications, vol. 19, no. 3, pp. 461-471, 2001.

[11] E. O. Ayoola, "Error estimates for discretized quantum stochastic differential inclusions," Stochastic Analysis and Applications, vol. 21, no. 6, pp. 1215-1230, 2003.

[12] E. O. Ayoola, "Topological properties of solution sets of Lipschitzian quantum stochastic differential inclusions," Acta Applicandae Mathematicae, vol. 100, no. 1, pp. 15-37, 2008.

[13] G. O. Ekhaguere, "Lipschitzian quantum stochastic differential inclusions," International Journal of Theoretical Physics, vol. 31, no. 11, pp. 2003-2027, 1992.

[14] R. L. Hudson and K. R. Parthasarathy, "Quantum Ito's formula and stochastic evolutions," Communications in Mathematical Physics, vol. 93, no. 3, pp. 301-323, 1984.

[15] G. O. S. Ekhaguere, "Quantum stochastic evolutions," International Journal of Theoretical Physics, vol. 35, no. 9, pp. 1909-1946, 1996. 


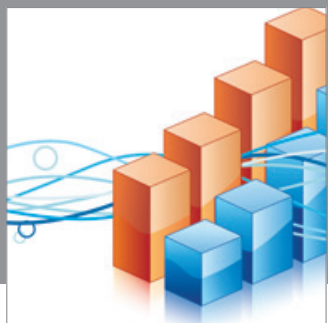

Advances in

Operations Research

mansans

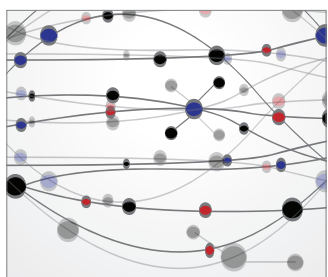

The Scientific World Journal
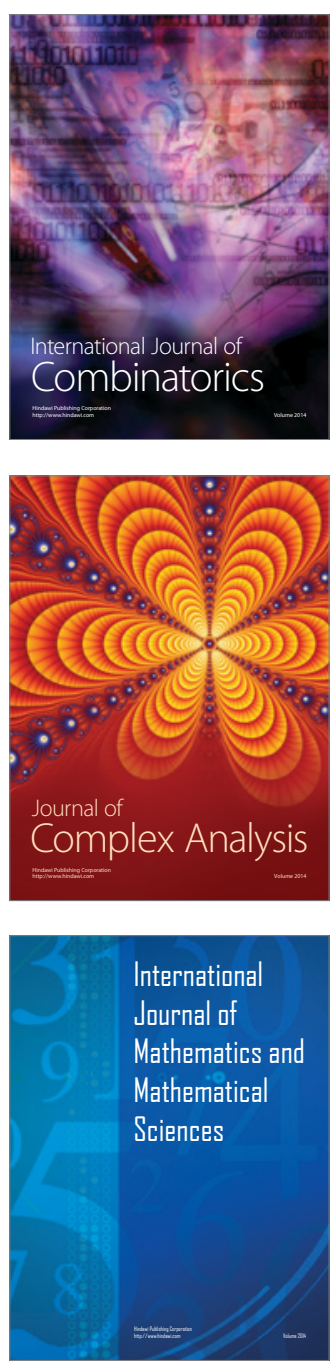
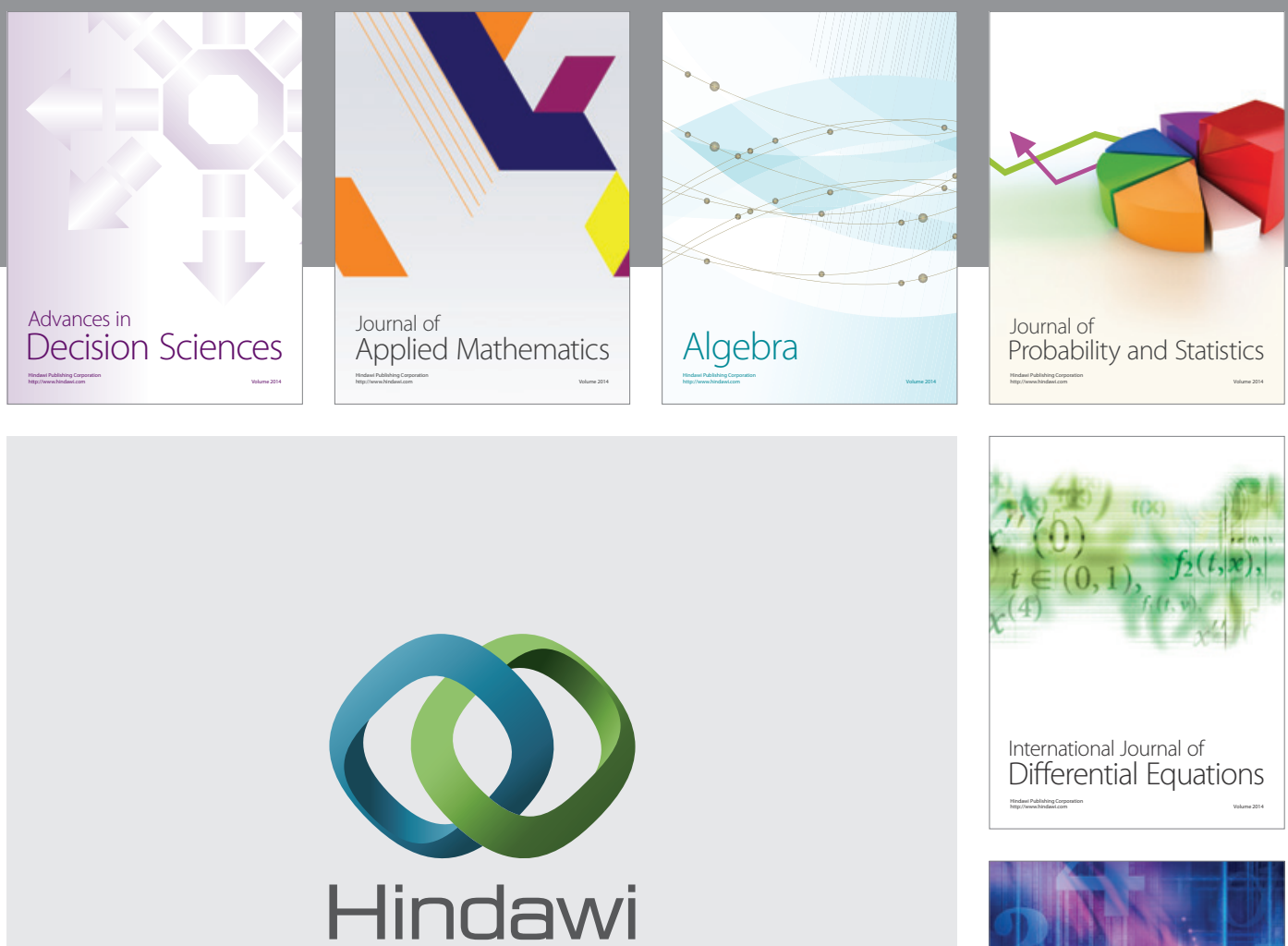

Submit your manuscripts at http://www.hindawi.com
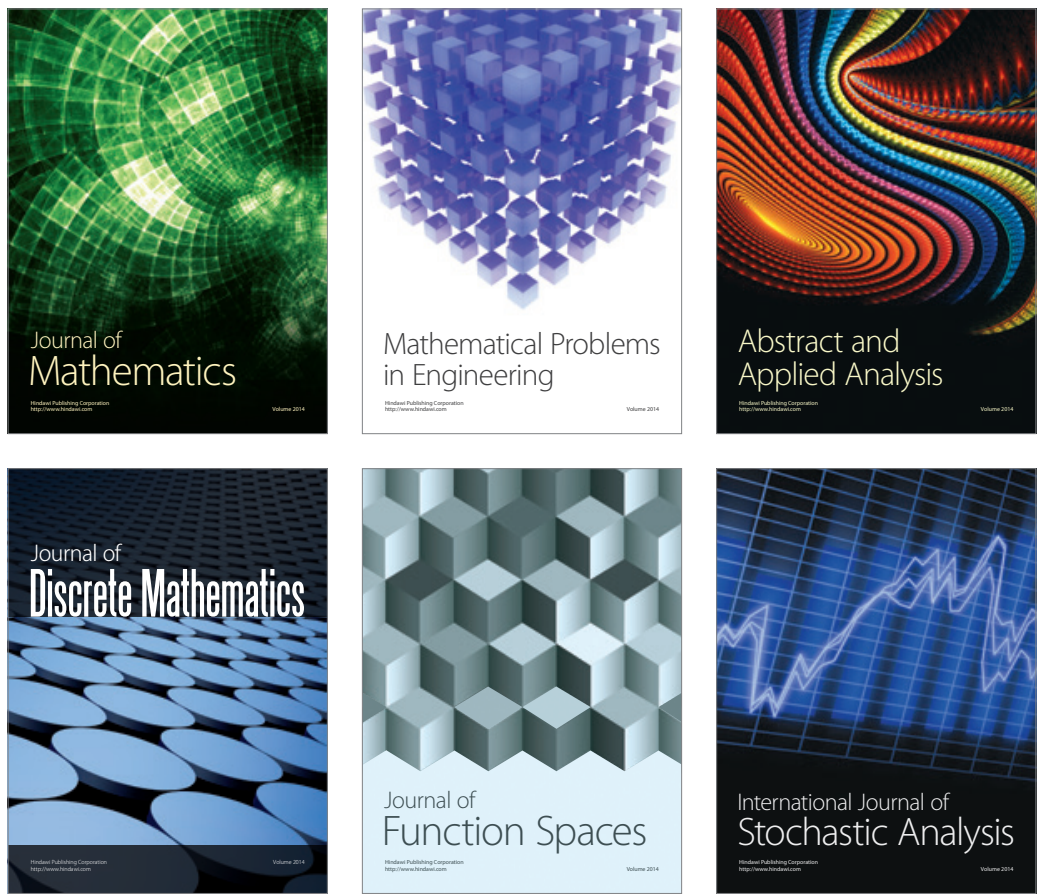

Journal of

Function Spaces

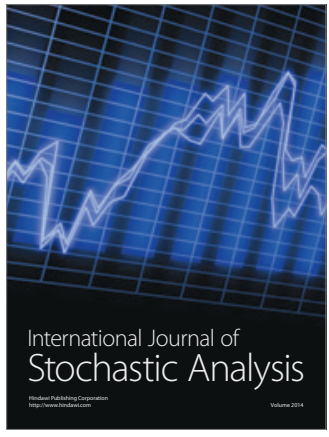

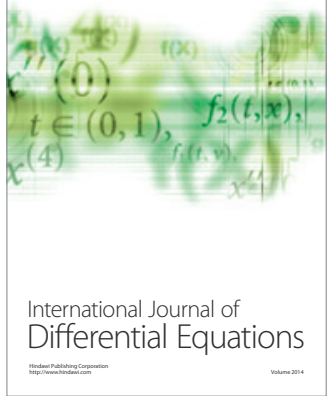
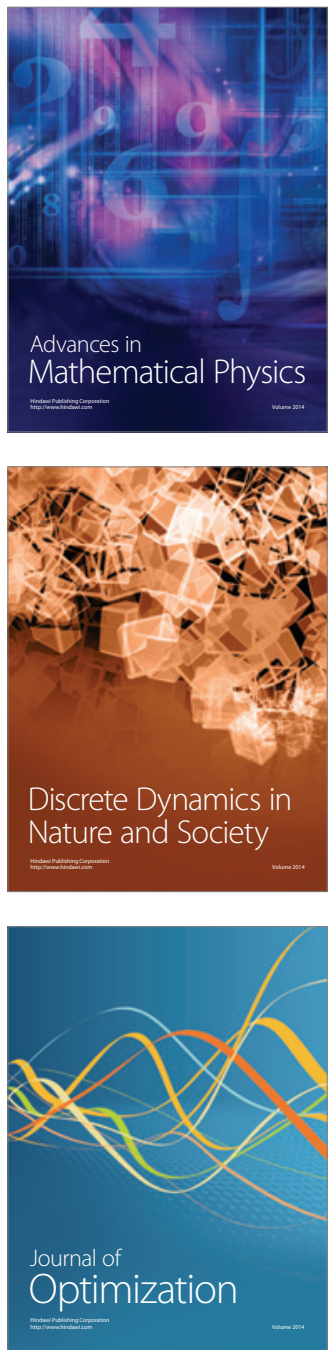